EUROPEAN ORGANIZATION FOR NUCLEAR RESEARCH

CERN - AB Department

CERN-AB-2005-042

\title{
TRANSVERSE EMITTANCE BLOW-UP DUE TO THE OPERATION OF WIRE SCANNERS, ANALYTICAL PREDICTIONS AND MEASUREMENTS
}

\author{
B. Dehning, F. Roncarolo
}

CERN, Geneva, Switzerland

\begin{abstract}
Wire Scanner monitors are used in the CERN accelerators to measure the transverse beam size. In the SPS and the LHC they will serve as calibration devices for other emittance monitors. The PSB, PS and SPS are equipped with scanners which move through the beam a 30 um wire through the beam, with a speed that can vary between 0.4 to $20 \mathrm{~m} / \mathrm{s}$. During each scan, the beam suffers an emittance blow up, due to multiple Coulomb scattering of the beam protons on the lattice nuclei of the wire material. The effect depends on the particles energy, the betatron function at the monitor location and on the monitor characteristics (wire material, diameter and speed). The comparaison between the predicted emittance increase and a number of experimental measurements is presented. For the small LHC beams the relative emittance blow-up can exceed several $10^{-2}$ in the SPS.
\end{abstract}

Presented at

PAC 2005, Knoxville, USA,

May 16-20, 2005

Geneva, Switzerland

June 2005 


\title{
Transverse Emittance Blow-Up Due to the Operation of Wire Scanners, Analytical Predictions and Measurements
}

\author{
F. Roncarolo, B. Dehning, CERN, Geneva, Switzerland
}

\begin{abstract}
Wire Scanner monitors are used in the CERN accelerators to measure the transverse beam size. In the SPS and the LHC they will serve as calibration devices for other emittance monitors. The PSB, PS and SPS are equipped with scanners which move a $30 \mu m$ wire through the beam, with a speed that can vary between 0.4 to $20 \mathrm{~m} / \mathrm{s}$. During each scan, the beam suffers an emittance blow up, due to multiple Coulomb scattering of the beam protons on the lattice nuclei of the wire material. The effect depends on the particles energy, the betatron function at the monitor location and on the monitor characteristics (wire material, diameter and speed). The comparison between the predicted emittance increase and a number of experimental measurements is presented. For the small LHC beams the relative emittance blow-up can exceed several $10^{-2}$ in the SPS.
\end{abstract}

\section{INTRODUCTION}

Multiple Coulomb scattering is described by Moliere's theory [1], which aims to study the angular distribution of the scattered particles. The distribution results roughly Gaussian for small scattering angles, although presents long non Gaussian tails below the $5 \%$ level. Many authors discussed Moliere's theory. Rossi and Greisen [2] provided a formula estimating the RMS value of the scattering angle projection on one plane orthogonal to the particles initial trajectory:

$$
\theta_{x}=\frac{15 \mathrm{MeV}}{\beta c p} z \sqrt{\frac{x}{L_{\text {rad }}}}
$$

in which $x$ is the material thickness and $\beta c, p$ and $z$ are the velocity, momentum and charge number of the incident particles. $L_{\text {rad }}$ is the radiation length of the material. Successive studies showed that Eq. (1) ignores significant dependencies from the particles path length and does not apply with the same accuracy to all materials. Highland [3] proposed a modified form of Eq. (1):

$$
\theta_{x}=\frac{17.5 \mathrm{MeV}}{\beta c p} z \sqrt{\frac{x}{L_{\text {rad }}}}\left[1+0.1 \log _{10}\left(\frac{10 x}{L_{\text {rad }}}\right)\right]
$$

This equation was based on analytical calculations and empirical interpolations to Moliere's theory. Eventually, Lynch and Dahl [4] compared the RMS scattering angle predictions given by Eq. (1) and Eq. (2) with the results of Monte Carlo simulations applying Moliere's theory. The comparison was done with different materials and ratios $x / L_{\text {rad }}$ varying from $10^{-3}$ to $10^{2}$. In their publication they remark that for $x / L_{\text {rad }}<10^{-3}$ Moliere's theory becomes less reliable, due to the very low number of scatters. The simulations include the case of $10^{-4} L_{\text {rad }}$ of Uranium, for which they reproduced a proper number of single scatters based on the classical Rutherford cross section. Lynch and Dahl's work lead to a new estimation of the RMS scattering angle,

$$
\theta_{x}=\frac{13.6 \mathrm{MeV}}{\beta c p} z \sqrt{\frac{x}{L_{\text {rad }}}}\left[1+0.038 \ln \left(\frac{x}{L_{\text {rad }}}\right)\right]
$$

valid for heavy charged particles with $\beta=1$ traversing any material. They asses the relative accuracy of Eq. (3) to $11 \%$ for $10^{-3}<x / L_{\text {rad }}<100$.

In the same publication, the two authors also formulated an estimation which is independent from the material radiation length. Such estimation has been later implemented in the Monte Carlo program named GEANT. Even if this program has not been used for any result presented in this paper, we introduce here the analytical relations adopted in the scattered particles distribution generator, since they will be used later:

$$
\begin{aligned}
\chi_{c c}^{2} & =\left(0.3961 \cdot 10^{-3}\right)^{2} Z \frac{\rho}{A}\left[\mathrm{GeV}^{2} \mathrm{~cm}^{-1}\right] \\
\theta_{x} & =2.557 \chi_{c c} \frac{\sqrt{x}}{p c \beta^{2}}
\end{aligned}
$$

where $\rho, A$ and $Z$ are the material density, atomic weight and charge number.

During measurements with very low emittance proton beams, a systematic emittance increase has been observed after the operation of the SPS wire scanners at a proton beam energy of $26 \mathrm{GeV}$. The increase is attributed to the scattering of the protons traversing the wire. Moliere's theory is not expected to be very accurate, since the used carbon wires diameter is only about $1.5 \cdot 10^{-4}$ in units of radiation length.

\section{THE SPS LINEAR WIRE SCANNERS}

In this paper we concentrate on the SPS linear WS monitors (that will also be adopted for LHC), for which the wire speed can be selected between 0.4 and $1 \mathrm{~m} / \mathrm{s}$. The monitors accuracy has been recently studied [6] by mean of beam based cross-calibrations. Fig. 1 shows a series of measurements with the TOTEM beam at $26 \mathrm{GeV}$, using the two linear WS monitors operating in the vertical plane. The two instruments have been operated simultaneously (with a time jitter of about $1 \mathrm{~ms}$ ), two times every filling cycle of the SPS. The histogram in Fig. 1(b) the mean reveals that the relative systematic discrepancy between the two instruments is below $1 \%$ in terms of normalized emittance. The repeatability of the difference (sigma on the plot) results 


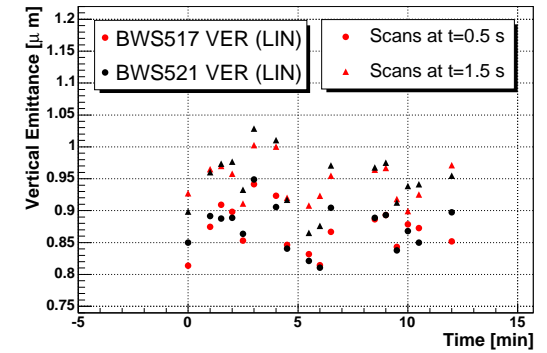

(a) Normalized vertical emittance as function of time

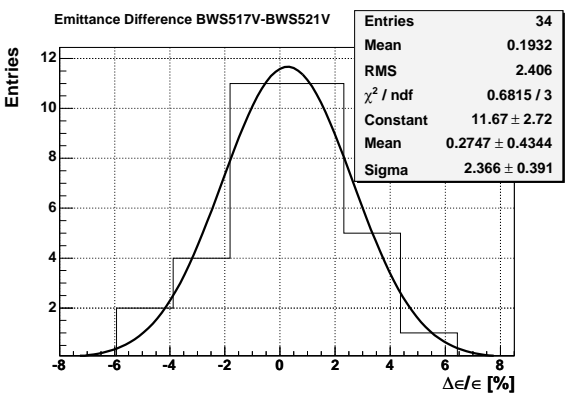

(b) Relative difference between the emittance as measured by the two monitors

Figure 1: Comparison between two linear WS during simultaneous measurements with the TOTEM beam injected every $30 \mathrm{~s}$ in the SPS.

better than $2.5 \%$, about $15 \mathrm{~nm}$ in terms of absolute normalized emittance.

\section{SIMULATIONS}

During a profile measurements with a WS monitor, at every particles revolution a number of protons encounters the carbon wire. Each of them has a probability of being scattered. The particles distribution is thus altered in both the transverse planes. Being the wire cross-section circular, an equivalent diameter $d_{e}$ has to be calculated. Formulating the locus of the points moving along the half circumference as $f(x)=\sqrt{r^{2}-x^{2}}$ with $-r<x<r$, where $r$ is the cross section radius, the equivalent diameter is found averaging over the cross section,

$$
d_{e}=\frac{2 \int_{-r}^{r} f(x) d x}{\int_{-r}^{r} d x}=\frac{2 \pi r^{2} / 2}{2 r}=\frac{\pi d}{4} .
$$

In the following the effects on one transverse plane are studied. The results are valid for both the horizontal and vertical planes, considering a monitor in a low dispersion region.

\section{Particles tracking}

A tracking simulation program has been developed. Starting from an arbitrary normalized emittance $\epsilon_{n}$, the initial Gaussian distribution of the particles in the phase space $\left(x, x^{\prime}\right)$ follows from the geometrical emittance $\epsilon_{x}=\epsilon_{n}$. $E_{0} /(E \beta)$ and the values of the Twiss parameters at the WS location. The initial wire position is set to $x_{w 0}=-5 \sigma_{0}$, where $\sigma_{0}=\sqrt{\beta_{x} \epsilon_{x}}$ is the initial beam size. The number of turns necessary for the tracking are determined by the time needed for the wire movement from $x_{w 0}$ to $x_{w 1}=+5 \sigma_{0}$. At every revolution period the one-turn transport matrix of the betatron motion is applied to the protons in the distribution and the wire moved of $\Delta x=v_{w} \cdot t_{r}$. A kick $\Delta x^{\prime}$ is applied to each particle with position $x$ such that $-d_{e} / 2<x<d_{e} / 2$. The kick amplitude is randomly extracted by a Gaussian distribution with width $\theta_{x}$ given by Eq. (3) or Eq. (5). At the same time the number of particles hitting the wire are used as amplitude signal of the profile which is turn-by-turn constructed as done during a real WS measurement.

The simulation of the WS profile during a tracking session is depicted in Fig. 2. The top plot shows the beam profile and the Gaussian fit. The bottom plot represents the residuals between the data and the fit, as function of position $x$. The initial distribution has been generated with a normalized emittance $\epsilon_{n}=0.5 \mu \mathrm{m}$ and according to the parameters at the location of the monitor labelled BWS517V: $\beta=100 m, \alpha=2$. The wire speed has been set to $v_{w}=1 \mathrm{~m} / \mathrm{s}$ (as it was set for the measurements that will be presented later) and 639 turns were necessary to complete the wire scan. Eq. (3) has been used to determine $\theta_{x}$. The beam size evolution, as calculated from the dis-

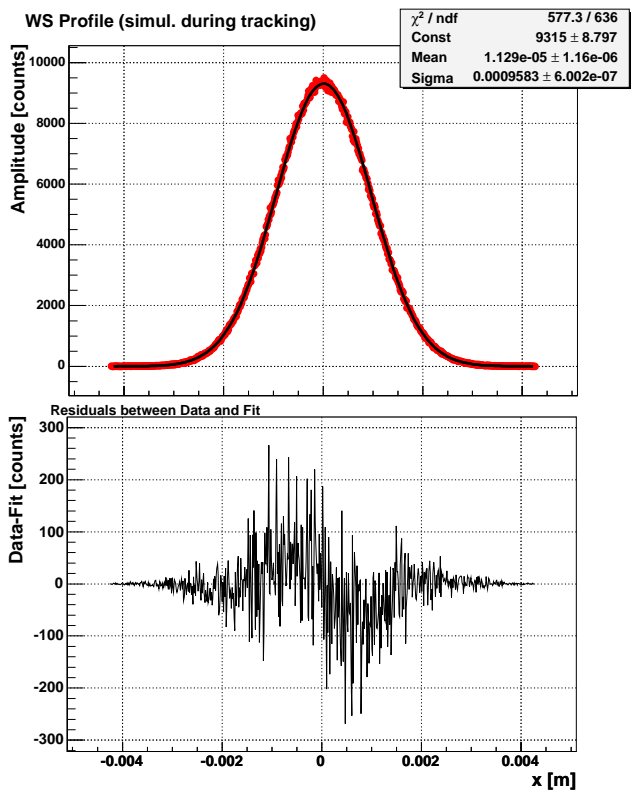

Figure 2: Simulation of the WS profile during the protons tracking.

tribution projections, is shown in Fig. 3 as function of the turn number. It increases from $0.948 \mathrm{~mm}$ to $0.966 \mathrm{~mm}$, while the Gaussian fit of the WS profile gives a beam size of $0.958 \mathrm{~mm}$. The WS only partially detects the emittance blow-up generated by the measurement itself. The effect 


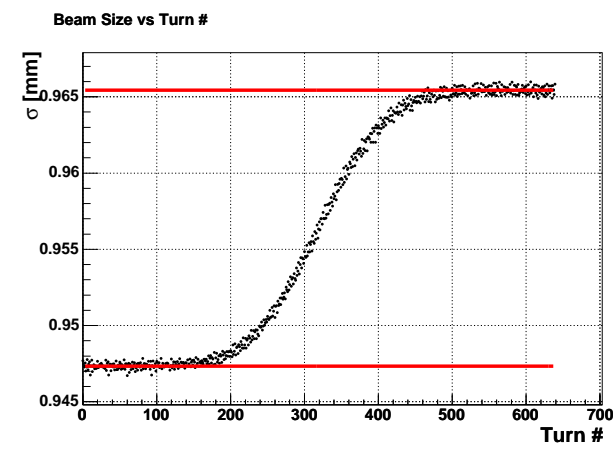

Figure 3: Beam size evolution during the tracking simulation example described in the text.

can be understood considering that the WS amplitude signal is acquired over a period during which the emittance is varying. The measured emittance results about half of the real final one.

The simulations have been carried out for initial normalized beam emittances varying from 0.2 to $2 \mu \mathrm{m}$ and showed that the emittance increase does not depends on the absolute beam size. The emittance increase generated by a wire scan measurement can also be predicted statistically treating the multiple Coulomb scattering of the particles populating the beam [7]. If $\sigma_{i}$ and $\sigma_{f}$ are the beam sizes before and after the scan, one finds

$$
\sigma_{f}^{2}-\sigma_{i}^{2}=P \frac{1}{2} \beta_{x}^{2} \theta_{x}^{2}
$$

where $\beta_{x}$ is the betatron function at the WS location. The quantity $P=d_{e} /\left(v_{w} t_{r}\right)$ represents the probability of a single particle to collide with the wire during a scan. The normalized emittance increase results:

$$
\Delta \epsilon_{n}=P \frac{1}{2} \beta_{x} \theta_{x}^{2} \cdot \frac{\beta E}{E_{0}}
$$

This prediction agrees to better than $1 \%$ with the simulation results.

\section{MEASUREMENTS IN THE SPS}

The systematic increase of the transverse emittance has been observed during measurements with two SPS linear wire scanners with TOTEM beam at $26 \mathrm{GeV}$. The blowup induced by the monitor labelled BWS517V is enhanced by its high betatron function $\left(\beta_{v}=100 \mathrm{~m}\right)$, while for BWS521V the low selected speed $(v=0.58 \mathrm{~m} / \mathrm{s})$ was critical . The emittance increase is measured by making the difference between two measurements with the same instrument during the same filling cycle. As an example see again Fig. 1(a). The beam size underestimation cancels in making the difference between two scans, which therefore results to be the emittance increase due to a single scan (plus the emittance increase due to a single scan of the other monitor, if used a the same time). The results of three different measurement sessions are presented in Table 1. During Period 1 both BWS517V and BWS517V were used,

\begin{tabular}{|l|c|c|c|c|}
\hline \multicolumn{5}{|c|}{ Emittance increase $\Delta \epsilon_{n}[\mathrm{~nm}]$} \\
\hline & Meas. & \multicolumn{3}{|c|}{ Predicted using: } \\
\hline & & Eq. (3) & $\begin{array}{c}\text { Eq. (3), } \\
\text { no log-term }\end{array}$ & Eq. (5) \\
\hline Period 1 & & 95 & 87 \\
\hline BWS517V & $79 \pm 4$ & 41 & 95 & 87 \\
BWS521V & $73 \pm 3$ & 41 & 51 & 47 \\
\hline Period 2 & \multicolumn{5}{|l|}{} \\
\hline BWS517V & $38 \pm 3$ & 22 & 44 & 40 \\
\hline Period 3
\end{tabular}

Table 1: Emittance increase as measured and predicted during three different periods (see text)

while during Period 2 and Period 3 the two instruments were operated singularly. The table also includes the emittance increase predictions when using Eq. (3) (with and without the logarithmic factor correction) or Eq. (5).

\section{CONCLUSIONS}

The comparison between the measurements and the approximations derived from the multiple Coulomb scattering theory shows that: i) the logarithmic correction factor proposed in Eq. (3), leads to an underestimation of the RMS scattering angle when used with $x / L_{\text {rad }} \approx 10^{-4}$; ii) Eq. (3) without logarithmic correction and Eq. (5) provide a maximum discrepancy with the measurements (in terms of normalized emittance increase) of 22 and $14 \mathrm{~nm}$ respectively when using the two instruments simultaneously; the discrepancies drop below $13 \mathrm{~nm}$ when only one monitor is operated. From predictions and measurements it can be concluded that a single scan of the considered devices at $26 \mathrm{GeV}$ induces a transverse emittance increase of the order of $50 \mathrm{~nm}$. This corresponds to about $5.5 \%$ of the TOTEM and $10 \%$ of the LHC pilot bunch emittances. About half of the increase is not detected during the measurement itself.

\section{Acknowledgments}

The authors would like to thank G. Arduini, M. Giovannozzi, S. Gilardoni and S. Redaelli for the very fruitful discussions on the subject.

\section{REFERENCES}

[1] G. Moliere, Z.Naturforsch 2a (1947) 133; 3a (1948) 78.

[2] B. Rossi and K. Greisen, Rev. Mod. Phys. 13 (1941)

[3] V.L. Highland, Nucl. Instr. and Meth. 129 (1975) 497.

[4] G.R. Lynch and O.I. Dahl, Nucl. Instr. and Meth. B58 (1991) 6.

[5] The European Physical Journal C, Springer, 1998.

[6] F. Roncarolo, Accuracy determination of the emittance monitors for LHC, PHD thesis, to be published.

[7] M. Syphers et al., Emittance Growth due to the Tevatron flying wires, FNAL, Beams-doc-1199-v2, 2004 